\title{
Lifetime improvement after phosphorous diffusion gettering on upgraded metallurgical grade silicon
}

\author{
Ana Peral ${ }^{\mathrm{a}^{*}}$, José Manuel Míguez ${ }^{\mathrm{b}}$, Ramón Ordás ${ }^{\mathrm{b}}$, and Carlos del Cañizo ${ }^{\mathrm{a}}$ \\ ${ }^{a}$ Instituto de Energía Solar, Universidad Politécnica de Madrid, Ciudad Universitaria, 28040 Madrid, Spain. \\ ${ }^{b}$ Ferrosolar (Grupo FerroAtlántica). Polígono Industrial el Sabón. Spain. \\ *Corresponding author e-mail address: ana.peral@ies-def.upm.es
}

\begin{abstract}
Wide experimental evidence of the phosphorus diffusion gettering beneficial effect on solar grade silicon is found by measuring electron effective lifetime and interstitial iron concentration in as-grown and post processed samples from two ingots of upgraded metallurgical grade silicon produced by Ferrosolar. Results after two different P-diffusion processes are compared: $\mathrm{P}$ emitter diffusion at $850^{\circ} \mathrm{C}$ followed by fast cool-down (called "standard process") or followed by slow cool-down (called "extended process"). It is shown that final lifetimes of this low cost material are in the range of those obtained with conventional material. The extended process can be beneficial for wafers with specific initial distribution and concentration of iron, e.g. materials with high concentration of big Fe precipitates, while for other cases the standard process is enough efficient. An analysis based on the comparison of measured lifetime and dissolved iron concentration with theoretical calculations helps to infer the initial iron distribution and concentration, and according to that, choose the more effective type of gettering.
\end{abstract}

Keywords: Solar grade Silicon, Upgraded Metallurgical Silicon, Phosphorus Diffusion Gettering

\section{Introduction}

P-type solar grade silicon produced by upgraded metallurgical route by Ferrosolar is a competitive low-cost feedstock material with an initial concentration of impurities higher than that of conventional polysilicon (typically $5-6 \mathrm{~N}$ vs. $9 \mathrm{~N}$ ) [1].

Through the thermal history of the solar cell, i.e. during the high temperature steps of the fabrication process, the distribution and concentration of the metal impurities vary and, consequently, their detrimental effect on the final material quality is modified. It is possible to reduce the impurity content during those steps, improving the final quality. The first high temperature step is the crystallization process, during which the silicon is purified in a 2 to 3 order of magnitude through solid-liquid segregation $[2,3]$. Consequently, the last solidified upper part of the mc-Si ingot is highly contaminated, and impurity back- and in-diffusion phenomena further deteriorate the upper, bottom and border regions of the ingot, respectively $[4,5]$.

The second high temperature step of the typical mc-Si solar cell fabrication chain is the Phosphorus Diffusion Gettering (PDG) at about $800-900{ }^{\circ} \mathrm{C}$ for several minutes. This step has been widely investigated and it has been highlighted that the gettering efficiency depends strongly on the as-grown iron concentration and distribution [6-8]. Some defect engineering tools have been developed recently based on this understanding to enhance the purification effect, such as the so-called extended gettering [911]. The phosphorus gettering efficiency using different temperature profiles has been successfully proved in solar-grade materials [12].

The purpose of this work is to study the effect of the phosphorus diffusion gettering process on this upgraded metallurgical grade (UMG) material, to compare the results of the standard PDG with defect engineering proposals such as the extended PDG, and to obtain a high quality material with lifetime comparable to the conventional one.

To reach this objective, extensive experimental work on samples coming from two ingots industrially produced by Ferrosolar is implemented. Measurements of minority carrier lifetime and interstitial Fe concentration before and after P-diffusion process help to characterize the material and the effect of the PDG process. Two temperature PDG profiles are implemented. P-diffusion gettering at $850{ }^{\circ} \mathrm{C}$ during 20 minutes is followed by: 1- a fast cool-down; 2- a slow exponential cool-down during 4 hours. Analysis of the results by fitting theoretical calculations with experimental measurements helps to understand $\mathrm{Fe}$ concentration and distribution into the as-grown wafers and then, based on this information, optimizing gettering temperature profile to reach higher final lifetime.

\section{Approach}

2.1 Material description

A set of 100 wafers (cut in samples of $4.5 \times 4.5 \mathrm{~cm}^{2}$ ) with a medium thickness of $200 \mu \mathrm{m}$ were analysed. They were taken from two p-type multicrystalline silicon ingots grown from upgraded 
metallurgical silicon produced by Ferrosolar. All wafers were selected from a center brick of the ingot (number 14). Wafers from Ingot 1 had a resistivity range from $0.6 \Omega \cdot \mathrm{cm}$ in the ingot middle to $1.5 \Omega \cdot \mathrm{cm}$ in the ingot top, as determined by four-point probe measurements. Wafers were selected from Ingot 1 heights $86 \%, 67 \%$ and $49 \%$. Resistivity of wafers from Ingot 2 varied between $1 \Omega \cdot \mathrm{cm}$ in the ingot bottom to $5 \Omega \cdot \mathrm{cm}$ in the ingot top. Ingot 2 wafers came from the ingot top and bottom.

\subsection{Experimental procedure}

All wafers have received the same process: a standard chemical cleaning, a $\mathrm{P}$ emitter diffusion at 850 ${ }^{\circ} \mathrm{C}$ during $20 \mathrm{~min}$ followed by a standard $\left(5 \mathrm{~min}\right.$ ) or extended (4 hours) temperature cool-down in $\mathrm{N}_{2}$ ambiance, a chemical etching to remove the $\mathrm{P}$ emitter, a standard chemical cleaning, and a PECVD deposition of silicon nitride on both sides for surface passivation. Finally, minority-carrier lifetime $(\tau)$ and interstitial iron concentration $\left(\left[\mathrm{Fe}_{\mathrm{i}}\right]\right)$ have been measured using the QSS-PC technique [13] in samples after standard PDG, and after extended PDG. At the same time, control wafers have been processed to characterize lifetime and $\left[\mathrm{Fe}_{\mathrm{i}}\right]$ on as-grown wafers.

2.3 Modelling iron impact on lifetime

Minority carrier lifetime limited by interstitial iron atoms associated to boron forming the well-known FeB pairs [14], $\tau_{F e B}$, is described by Shockley-Read-Hall statistics under low injection conditions [15, 16]:

$$
\frac{1}{\tau_{F e B}}=[F e B] \sigma v_{t h}
$$

where $\sigma$ is the minority carrier capture cross section, $v_{t h}$ is the thermal velocity of minority carriers, and $[\mathrm{FeB}]$ is concentration of Fe-B pairs.

Precipitate-limited lifetime, $\tau_{p}$, is described by the following expression [17]:

$$
\frac{1}{\tau_{p}}=4 \pi r^{2} \frac{\left[F e_{p}\right] V_{F e}}{\frac{4}{3} \pi r^{3}} \frac{s D / r}{s+D / r}
$$

where $r$ is the precipitate radius, $\left[F e_{p}\right]$ is the precipitated iron concentration, $V_{F e}$ is the Fe atom volume, $D$ is the minority carrier diffusion length, and $s$ is the carrier recombination velocity at the precipitate surface [18].

Then, effective lifetime, $\tau$, can be calculated as:

\section{Results and discussion}

$$
\frac{1}{\tau}=\frac{1}{\tau_{F e B}}+\frac{1}{\tau_{p}}
$$

\subsection{Experimental results}

Minority-carrier lifetime measurements of wafers from top and middle of Ingot 1 and from top and bottom of Ingot 2 are shown in Figure 1. Diamonds, circles and crosses correspond to as-grown, post standard PDG and post extended PDG measurements, respectively. Interstitial iron concentration measurements from the same samples are shown in Figure 2.

As can be seen from Fig. 1, as-grown samples present lifetime measurements with values below 10 $\mu$ s. In addition, we observe a slightly decrease of as-grown lifetime with increasing ingot height. Lifetime measurements of samples from ingot top are slightly smaller than as-grown measurements of samples from the middle and bottom of the ingot $\left(\tau_{\text {Ingot1_Top }}=7-8 \mu \mathrm{s}, \tau_{\text {Ingot1_Middle }}=8-11 \mu \mathrm{s}, \tau_{\text {Ingot2_Top }}=1-6 \mu \mathrm{s}\right.$, and $\tau_{\text {Ingot2_Bottom }}=5-9 \mu \mathrm{s}$ ). Both post standard and extended P-diffusion gettering lifetime measurements increase above the as-grown value. Gettering efficiency is higher with increasing ingot height.

As-grown samples from top of Ingot 1 (see Fig. 2) present a mean interstitial iron concentration higher than middle wafers $\left(\left[\mathrm{Fe}_{\mathrm{i}}\right]_{\text {Ingot1_Top }}=2 \cdot 10^{12} \mathrm{~cm}^{-3}\right.$, and $\left.\left[\mathrm{Fe}_{\mathrm{i}}\right]_{\text {Ingot1_Middle }}=8 \cdot 10^{11} \mathrm{~cm}^{-3}\right)$. However, Ingot 2 presents a mean dissolved iron concentration in top wafers lower than bottom wafers $\left(\left[\mathrm{Fe}_{\mathrm{i}}\right]_{\text {Ingot2_Top }}=\right.$ $1.5 \cdot 10^{11} \mathrm{~cm}^{-3}$, and $\left.\left[\mathrm{Fe}_{\mathrm{i}}\right]_{\text {Ingot2_Bottom }}=5 \cdot 10^{11} \mathrm{~cm}^{-3}\right)$. Post standard PDG mean $\left[\mathrm{Fe}_{\mathrm{i}}\right]$ measurements decrease below as-grown values for samples from Ingot 1 and bottom of Ingot 2. On the contrary, samples from the top of Ingot 2 have a post standard PDG mean $\left[\mathrm{Fe}_{\mathrm{i}}\right]$ higher than as-grown value. Post extended PDG mean $\left[\mathrm{Fe}_{\mathrm{i}}\right]$ measurements decrease below as-grown values and below post standard PDG values for wafers from Ingot 1 and bottom of Ingot 2. However, post extended PDG [Fe $\mathrm{F}_{\mathrm{i}}$ measurements of samples from top of Ingot 2 increase above as-grown values and above post standard PDG values. 


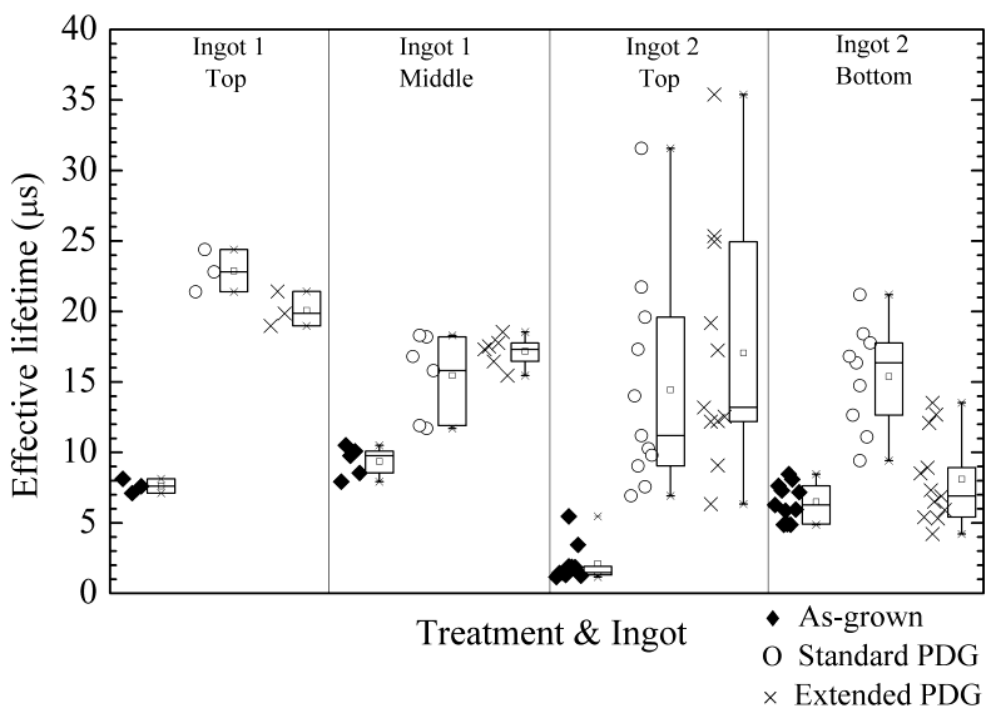

Figure 1 Minority-carrier lifetime measurements of samples as-grown (diamonds), post standard PDG (circles) and post extended PDG (crosses). Wafers come from two UMG ingots provided by Ferrosolar.

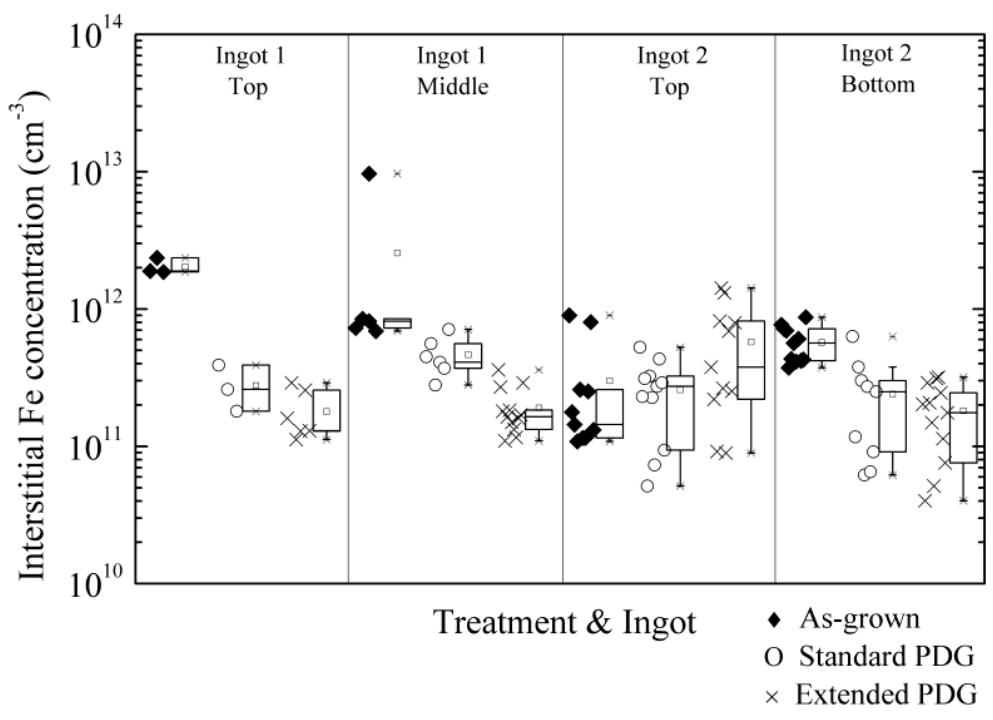

Figure 2 Interstitial iron concentration measurements of samples as-grown (diamonds), post standard PDG (circles) and post extended PDG (crosses). Wafers come from two UMG ingots provided by Ferrosolar.

\subsection{Simulations and discussion}

We represent as-grown experimental lifetime as a function of measured interstitial iron concentration (each point in Figure 3 (a) correspond to a wafer lifetime measurement). Samples from top and middle of Ingot 1 are plotted as squares and triangles respectively, and samples from top and bottom of Ingot 2 are plotted as crosses and circles respectively.

Then we fit theoretical curves of effective lifetime (Eq. 3) with the experimental measurements. As result of the fitting, each experimental measurement of lifetime-[Fe $\left.\mathrm{Fe}_{\mathrm{i}}\right]$ is related to a total iron concentration $\left(\left[\mathrm{Fe}_{\mathrm{T}}\right]\right)$ and distribution (i.e. radius).

As can be seen from Fig. 3 (a), we can distinguish three as-grown material types depending on their total iron concentration and distribution:

1. Materials from Ingot 1 (squares and triangles): Their lifetime-[Fei] measurements correspond to a material with an effective lifetime limited by Fe-B pairs, plotted with a solid line. Also, measurements may correspond to a material with an effective lifetime limited by a low concentration of very small Fe precipitates, plotted with a dash line (for effective lifetime calculations, a total Fe concentration of $1.9 \cdot 10^{13} \mathrm{~cm}^{-3}$ and a radius precipitate of $8 \mathrm{~nm}$ has been assumed). This value of total Fe concentration is in agreement with that resulting from applying 
Scheil equation to the total Fe measured by ICP-MS in the feedstock (3.8 ppmw); with an effective segregation coefficient of iron in DS grown mc-Si equal to $1.6 \cdot 10^{-4}$ [2], Fe concentration ranges between $2 \cdot 10^{13}$ and $1 \cdot 10^{14} \mathrm{~cm}^{-3}$ along the middle and top ingot heights.

2. Materials from the bottom of Ingot 2 (circles): Their lifetime-[Fei] measurements correspond to a material with an effective lifetime limited by a medium concentration of small Fe precipitates, plotted with a dot line (for effective lifetime calculations, a total Fe concentration of $2 \cdot 10^{14} \mathrm{~cm}^{-3}$ and a radius precipitate of $15 \mathrm{~nm}$ has been assumed).

3. Materials from the top of Ingot 2 (crosses): Their lifetime-[Fei] measurements correspond to a material with an effective lifetime limited by a high concentration of big Fe precipitates, plotted with a dash-dot line (for effective lifetime calculations, a total Fe concentration of $5 \cdot 10^{15} \mathrm{~cm}^{-3}$ and a radius precipitate of $30 \mathrm{~nm}$ has been assumed).

According to this, it can be observed that with ingot height increment, both total Fe concentration and Fe precipitate radius increase. Similar conclusions have been reached by Fenning et al. [19].

The evolution of total iron concentration and Fe precipitate size after P-diffusion would depend on the initial state (see Fig. 3 b and c):

1. Materials from Ingot 1 (squares and triangles):

- After standard PDG, they could be limited by a low concentration of very small precipitates or by $\mathrm{Fe}-\mathrm{B}$ (solid line and dash line respectively).

- After extended PDG, lifetime could be limited by other impurities or defects different from FeB. Then, slow cool-down does not help to increase lifetime, although interstitial iron concentration has decreased.

2. Materials from the bottom of Ingot 2 (circles):

- After standard PDG, their lifetime could be limited by a medium concentration of small precipitates or by Fe-B. Both total Fe concentration and radius size may have been reduced.

- After extended PDG, they could be limited by a medium concentration of small precipitates or by $\mathrm{Fe}-\mathrm{B}$. The slow cooling reduces interstitial $\mathrm{Fe}$ concentration however it does not help to increase lifetime.

3. Materials from the top of Ingot 2 (crosses):

- After standard PDG, they could be limited by a medium concentration of small precipitates or by $\mathrm{Fe}-\mathrm{B}$. Both total $\mathrm{Fe}$ concentration and radius size has been reduced.

- After extended PDG, they could be limited by a low concentration of very small precipitates or by $\mathrm{Fe}-\mathrm{B}$. The slow cooling may reduce iron precipitate radius size and total Fe concentration.

From the comparison of post P-diffusion measurements with theoretical calculations, it can be observed that, on the one hand, standard PDG can reduce both total Fe concentration and precipitate radius; and, on the other hand, extended PDG slow cooling can increase lifetime in materials that have post standard PDG lifetime limited by $\mathrm{Fe}$ precipitates (i.e. Ingot 2 Top), by reducing both total $\mathrm{Fe}$ concentration and precipitate radius. However, it would be enough to implement a standard PDG in materials with a post standard PDG lifetime limited by small precipitates or by Fe-B (i.e. Ingot 1 and Ingot 2 Bottom). 
As-grown measurements

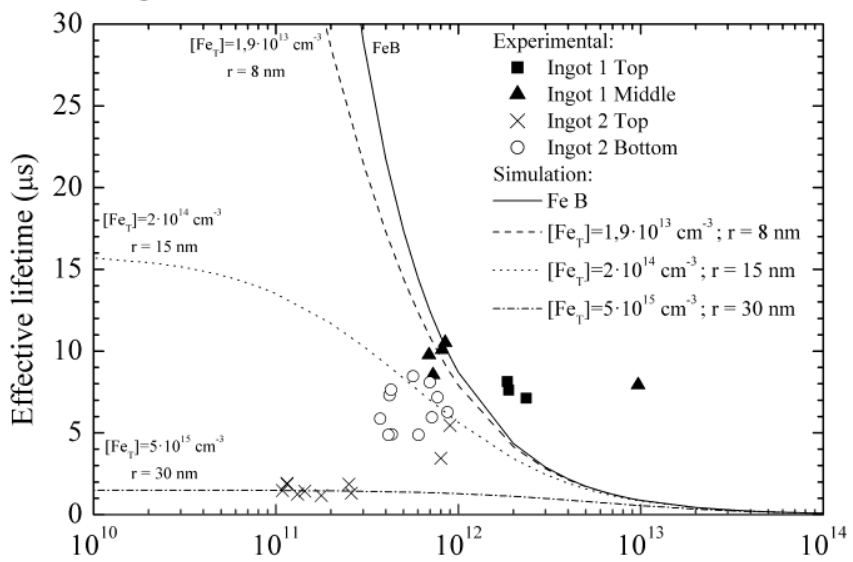

Interstitial Fe concentration $\left(\mathrm{cm}^{-3}\right)$

(a)

Post standard PDG measurements

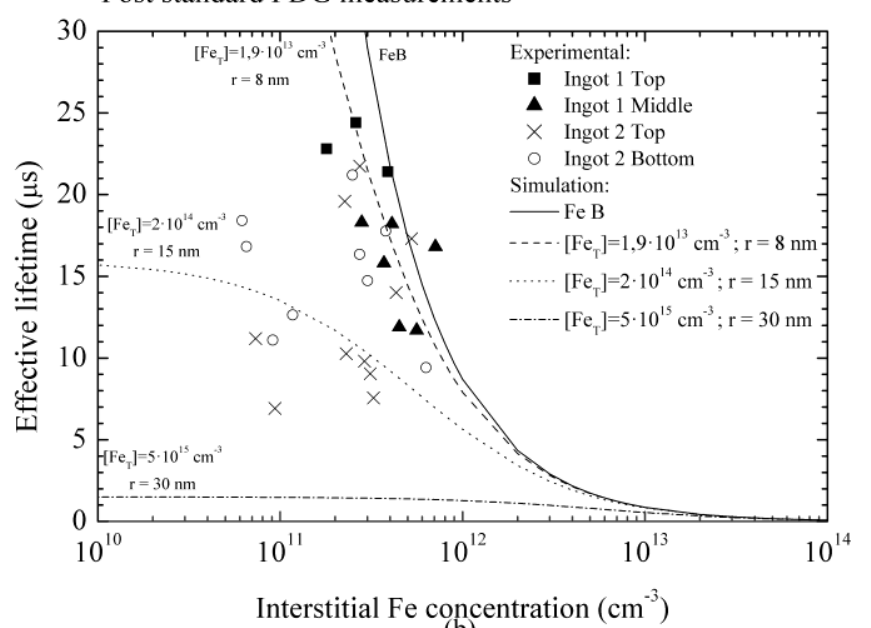

(b)

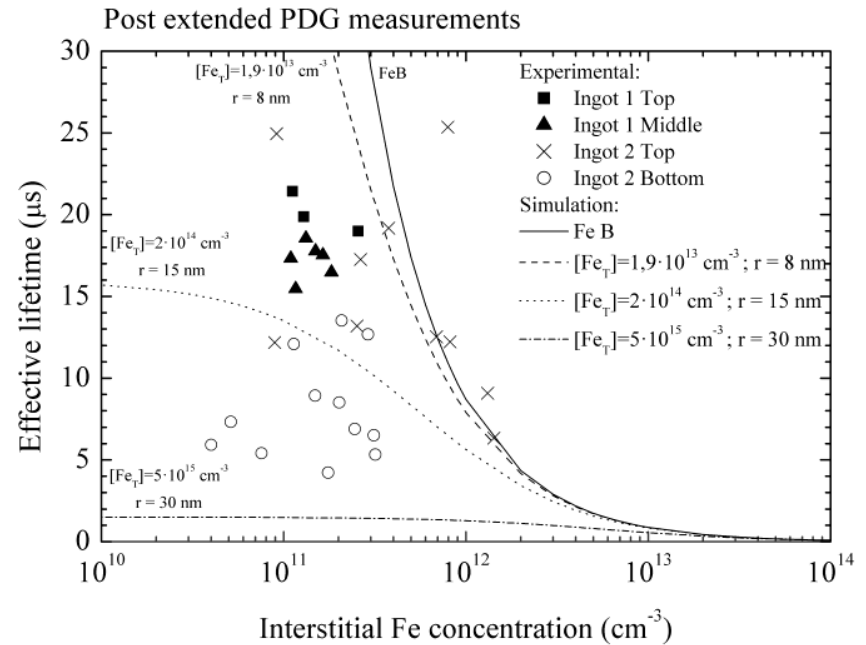

(c)

Figure 3 Electron lifetime as a function of the interstitial iron concentration measured on samples from top and middle of Ingot 1 (squares and triangles respectively) and from top and bottom of Ingot 2 (crosses and circles respectively); as-grown measurements are showed in (a), post standard PDG in (b) and post extended PDG in (c); the calculated electron lifetime as a function of the Fe-B pair concentration shown as solid line; the other lines show the effective lifetime as a function of the dissolved iron concentration in the case of having different total iron concentrations 


\section{Conclusions}

Phosphorus Diffusion Gettering is shown to be effective for the low-cost solar grade silicon produced by Ferrosolar, giving final lifetimes comparable with those of conventional material.

Initial material lifetime can be potentially limited by dissolved iron or precipitated iron. Experimental results show that depending on the initial Fe concentration and distribution, it is worth to implement an extended gettering, which may increase final lifetime (e.g. for the wafers containing high concentration of big precipitates) or, on the contrary, implementing the standard one may be enough for obtaining high lifetime experimental measurements (e.g. for wafers containing small concentration of small precipitates).

A hint on the initial iron concentration and distribution can be inferred by fitting theoretical calculations with usual measurements of dissolved iron concentration and lifetime on as-grown wafers, helping then to choose the more effective type of gettering to be applied to the material.

More different cases of initial iron concentration and distribution, as well as other P-gettering temperature profiles optimized as a function of this initial contamination, will be included in future works and will help to find strategies to improve carrier lifetime of UMG-Si materials.

\section{Acknowledgements}

This work has been partially funded by the Spanish Ministerio de Economía y Competitividad, through Crysthin (TEC2011-28423-C03) project.

\section{References}

[1] J.M. Míguez, J. Bullón, R. Ordás, T. Carballo, J. Diéguez, A. Miranda, O. Martínez, A. Souto, J. Jiménez, V. Parra, A. Pérez, B. Moralejo, UMG-Si based PV materials and devices: current state and future trends, in: Proceedings of the 6th International Workshop on Crystalline Silicon Solar Cells, 6.O.1, Aix-les-Bains, France, 2012.

[2] S. Pizzini, M. Acciarri, S. Binetti, From electronic grade to solar grade silicon: chances and challenges in photovoltaics, Phys. Status Solidi A, 202 (2005) 2928-2942.

[3] J. Hofstetter, J.F. Lelievre, C. del Canizo, A. Luque, Acceptable contamination levels in solar grade silicon: From feedstock to solar cell, Mater. Sci. Eng. B, 159-160 (2009) 299-304.

[4] D. Macdonald, A. Cuevas, A. Kinomura, Y. Nakano, L.J. Geerligs, Transition-metal profiles in a multicrystalline silicon ingot, J. Appl. Phys., 97 (2005) 033523.

[5] T.U. Nærland, L. Arnberg, A. Holt, Origin of the low carrier lifetime edge zone in multicrystalline PV silicon, Prog. Photovolt: Res. Appl., 17 (2009) 289-296.

[6] J.S. Kang, D.K. Schroder, Gettering in silicon, J. Appl. Phys., 65 (1989) 2974-2985.

[7] J. Hofstetter, D.P. Fenning, M.I. Bertoni, J.F. Lelièvre, C. del Cañizo, T. Buonassisi, Impurity-toefficiency simulator: predictive simulation of silicon solar cell performance based on iron content and distribution, Prog. Photovolt: Res. Appl., 19 (2011) 487-497.

[8] M. Syre, S. Karazhanov, B.R. Olaisen, A. Holt, B.G. Svensson, Evaluation of possible mechanisms behind P gettering of iron, J. Appl. Phys., 110 (2011) 024912.

[9] T. Buonassisi, A.A. Istratov, M.A. Marcus, B. Lai, Z. Cai, S.M. Heald, E.R. Weber, Engineering metal-impurity nanodefects for low-cost solar cells, Nature Materials, 4 (2005) 676-679.

[10] M. Rinio, A. Yodyunyong, S. Keipert-Colberg, Y.P.B. Mouafi, D. Borchert, A. MontesdeocaSantana, Improvement of multicrystalline silicon solar cells by a low temperature anneal after emitter diffusion, Prog. Photovolt: Res. Appl., 19 (2011) 165-169.

[11] J. Hofstetter, J.-F. Lelièvre, D.P. Fenning, M.I. Bertoni, T. Buonassisi, A. Luque, C. del Cañizo, Enhanced iron gettering by short, optimized low-temperature annealing after phosphorus emitter diffusion for industrial silicon solar cell processing, Phys. Status Solidi C, 8 (2011) 759-762.

[12] V. Osinniy, A. Nylandsted Larsen, E. Hvidsten Dahl, E. Enebakk, A.K. Søiland, R. Tronstad, Y. Safir, Gettering improvements of minority-carrier lifetimes in solar grade silicon, Sol. Energ. Mat. Sol. Cells, 101 (2012) 123-130.

[13] D.H. Macdonald, L.J. Geerligs, A. Azzizi, Iron detection in crystalline silicon by carrier lifetime measurements for arbitrary injection and doping, J. Appl. Phys., 95 (2004) 1021-1028.

[14] K. Graff, Metal Impurities in Silicon-Device Fabrication, Springer Series in Materials Science 1995.

[15] W. Shockley, W.T. Read, Statistics of the Recombinations of Holes and Electrons, Phys. Rev., 87 (1952) 835-842.

[16] R.N. Hall, Electron-Hole Recombination in Germanium, Phys. Rev., 87 (1952) 387-387.

[17] C. del Cañizo, A. Luque, A Comprehensive Model for the Gettering of Lifetime-Killing Impurities in Silicon, Journal of The Electrochemical Society, 147 (2000) 2685-2692. 
[18] P.S. Plekhanov, T.Y. Tan, Schottky effect model of electrical activity of metallic precipitates in silicon, Appl. Phys. Lett., 76 (2000) 3777-3779.

[19] D.P. Fenning, J. Hofstetter, M.I. Bertoni, G. Coletti, B. Lai, C. del Cañizo, T. Buonassisi, Precipitated iron: A limit on gettering efficacy in multicrystalline silicon, J. Appl. Phys., 113 (2013). 